\title{
Spectra inhabiting the left half-plane that are universally realizable
}

https://doi.org/10.1515/spma-2021-0155

Received September 31, 2021; accepted December 5, 2021

Abstract: Let $\Lambda=\left\{\lambda_{1}, \lambda_{2}, \ldots, \lambda_{n}\right\}$ be a list of complex numbers. $\Lambda$ is said to be realizable if it is the spectrum of an entrywise nonnegative matrix. $\Lambda$ is universally realizable if it is realizable for each possible Jordan canonical form allowed by $\Lambda$. Minc ([21],1981) showed that if $\Lambda$ is diagonalizably positively realizable, then $\Lambda$ is universally realizable. The positivity condition is essential for the proof of Minc, and the question whether the result holds for nonnegative realizations has been open for almost forty years. Recently, two extensions of the Minc's result have been proved in ([5], 2018) and ([12], 2020). In this work we characterize new left halfplane lists $\left(\lambda_{1}>0, \operatorname{Re} \lambda_{i} \leq 0, i=2, \ldots, n\right)$ no positively realizable, which are universally realizable. We also show new criteria which allow to decide about the universal realizability of more general lists, extending in this way some previous results.

Keywords: Nonnegative matrix, universal realizability, Jordan structure

MSC: 15A18, 15A29

\section{Introduction}

A list $\Lambda=\left\{\lambda_{1}, \lambda_{2}, \ldots, \lambda_{n}\right\}$ of complex numbers is said to be realizable, if it is the spectrum of an $n$-by- $n$ nonnegative matrix $A$. In this case, $A$ is said to be a realizing matrix. Since that any list of complex numbers is the spectrum of some matrix (trivially a diagonal matrix), we will sometimes use the word spectrum instead of the word list. The problem of the realizability of spectra is called Nonnegative inverse eigenvalue problem (NIEP). A spectrum $\Lambda$ is said to be universally realizable (UR), if it is realizable for every possible Jordan canonical form (JCF) allowed by $\Lambda$. The problem of determining the universal realizability of spectra, which is the subject of this work, is called Universal realizability problem (URP). We shall say that $\Lambda=\left\{\lambda_{1}, \lambda_{2}, \ldots, \lambda_{n}\right\}$ is a left half-plane spectrum or that $\Lambda$ inhabits the left half-plane if $\lambda_{1}>0$, and $\operatorname{Re} \lambda_{i} \leq 0, i=2,3, \ldots, n$.

The NIEP originates from results of Kolmogorov ([17], 1937) and Karpelevic ([16], 1951). However, as we know it today, begins with the works of Suleimanova ([34], 1949) and Perfect ([23], 1953 and [24], 1955). The NIEP contains two important sub-problems: the Real nonnegative inverse eigenvalue problem (RNIEP), in which the given list $\Lambda=\left\{\lambda_{1}, \lambda_{2}, \ldots, \lambda_{n}\right\}$ admits only real numbers, and the Symmetric nonnegative inverse eigenvalue problem (SNIEP), in which a realizing matrix is required to be symmetric. The NIEP has been solved only for the cases $n=3$, by Loewy and London [19], and $n=4$, by Meehan [20], and independently by Torre-Mayo et.al. [35], which shows the difficulty of the problem. The case $n=5$ zero trace was solved by Laffey and Meehan [18], while the SNIEP, $n=5$ zero trace was recently solved by Spector [33].

For $n \geq 5$ both problems, the RNIEP and the SNIEP, are different [10], and remain unsolved. A number of necessary conditions and sufficient conditions are known in the literature about them. However, there is still

`Corresponding Author: Ricardo L. Soto: Departamento de Matemáticas, Universidad Católica del Norte, Antofagasta, Chile, Casilla 1280, Antofagasta, Chile, E-mail: rsoto@ucn.cl 
a big gap between necessary conditions and sufficient conditions: necessary conditions are too general, while sufficient conditions are too specific. For an elaborate exposition on the history of the NIEP we refer the reader to [11].

From the Perron-Frobenius Theorem, if $\Lambda=\left\{\lambda_{1}, \lambda_{2}, \ldots, \lambda_{n}\right\}$ is the spectrum of an $n$-by- $n$ nonnegative matrix $A$, then the leading eigenvalue of $A$ equals to the spectral radius of $A$, namely $\rho(A)=: \max _{1 \leq i \leq n}\left|\lambda_{i}\right|$. This eigenvalue is called the Perron eigenvalue, and we shall assume in this paper, that $\rho(A)=\lambda_{1}$. An $n$-by- $n$ matrix $A=\left(a_{i j}\right)$ is said to have constant row sums, if all its rows sum a same constant, say $\alpha$. We denote by $\mathcal{C} \mathcal{S}_{\alpha}$ the set of all $n$-by- $n$ real matrices with constant row sums $\alpha$. It is clear that any matrix in $\mathcal{C S}_{\alpha}$ has an eigenvector $\mathbf{e}=[1, \ldots, 1]^{T}$ corresponding to the eigenvalue $\alpha$. The relevance of the real matrices with constant row sums, is due to the fact that the problem of finding a nonnegative matrix with spectrum $\Lambda=\left\{\lambda_{1}, \lambda_{2}, \ldots, \lambda_{n}\right\}$, is equivalent to the problem of finding a nonnegative matrix in $\mathcal{E} \mathcal{S}_{\lambda_{1}}$ with spectrum $\Lambda$ [9].

The URP and the NIEP are closely related. The URP contains the NIEP, and both problems are equivalent if the given complex numbers, $\lambda_{1}, \lambda_{2}, \ldots, \lambda_{n}$, are distinct. The first known results on the URP are due to Minc [21, 22]. Minc ([21],1981) shows that if a list $\Lambda=\left\{\lambda_{1}, \lambda_{2}, \ldots, \lambda_{n}\right\}$ of complex numbers is diagonalizably positively realizable, then $\Lambda$ is universally realizable. The positivity condition in the result of Minc [21] is essential for his proof and the question whether the result holds for nonnegative realizations was open for almost 40 years. Recently, two extensions of the Minc's result were obtained by Collao, Salas and Soto [5], and by Johnson, Julio and Soto [12]. They answer positively the above question. In particular, in [5], the authors prove that if a list $\Lambda$ of complex numbers is the spectrum of a nonnegative diagonalizable matrix $A \in \mathcal{C S}_{\lambda_{1}}$ with a positive row or column, then $\Lambda$ is UR. In [12], the authors prove that if a list $\Lambda$ of complex numbers is diagonalizably ODP realizable, that is, if $\Lambda$ is the spectrum of a diagonalizable nonnegative matrix with only off-diagonal positive entries (zero entries are allowed on the diagonal), then $\Lambda$ is also UR. For instance, spectra of normal ODP, circulants ODP, symmetric ODP, bisymmetric ODP, between other are UR. Observe that the set of ODP matrices contain the set of positive matrices. In $[7,27,29]$ the authors prove, respectively, that there are spectra nonpositively realizable, which are universally realizable. That is, they prove that the following lists are all UR if and only if $\sum_{i=1}^{n} \lambda_{i} \geq 0$ :

i) [27], lists of real numbers $\Lambda=\left\{\lambda_{1}, \lambda_{2}, \ldots, \lambda_{n}\right\}$ with

$$
\lambda_{1}>0>\lambda_{2} \geq \lambda_{3} \geq \cdots \geq \lambda_{n},
$$

ii) [29], lists of complex numbers $\Lambda=\left\{\lambda_{1}, \lambda_{2}, \ldots, \lambda_{n}\right\}$ with $\lambda_{1}>0$ and

$$
\lambda_{i} \in \mathcal{F}=\{z \in \mathbb{C}: \operatorname{Re} z \leq 0,|\operatorname{Re} z| \geq|\operatorname{Im} z|\}, i=2, \ldots, n,
$$

iii) [7], lists of complex numbers $\Lambda=\left\{\lambda_{1}, \lambda_{2}, \ldots, \lambda_{n}\right\}$ with $\lambda_{1}>0$ and

$$
\lambda_{i} \in \mathcal{G}=\{z \in \mathbb{C}: \operatorname{Re} z \leq 0,|\sqrt{3} \operatorname{Re} z| \geq|\operatorname{Im} z|\}, i=2, \ldots, n .
$$

Others results, in terms of sufficient conditions or realizability criteria for a solution to the URP, can be found in $([4,5,12,14,15,30,31]$ and the references therein)

Despite the progress that has been made in relation to the URP, there are still numerous open questions. For example, we would like to know if in general, diagonalizably realizable implies universally realizable. In [13] a negative answer was given in the case of reducible realizations, but we do not know the answer in the case of irreducible realizations. Is any realizable list in the left half-plane UR? In [15] the authors show that there are lists inhabiting the left half-plane, which are realizable, but not UR. Then, to what extension this kind of lists are UR? Is the spectrum of a nonnegative structured matrix, UR with the same structure? In this work we characterize new lists in the left half-plane, which are universally realizable. We also give new results, which allow to decide about the universal realizability of more general lists of complex numbers, and we improve previous criteria. 
The following perturbation result, due to Rado and published by Perfect in [24], has been important to generate many realizability criteria known in the literature about the NIEP and the URP. It shows how to change $r$ eigenvalues of an $n$-by- $n$ matrix, without changing any of the remaining $n-r$ eigenvalues.

Theorem 1.1. [24] Rado. Let $A$ be an $n$-by-n matrix with spectrum $\Lambda=\left\{\lambda_{1}, \ldots, \lambda_{n}\right\}$. Let $X=\left[\mathbf{x}_{1}|\cdots| \mathbf{x}_{r}\right]$ such that $\operatorname{rank}(X)=r$ and $A \mathbf{x}_{i}=\lambda_{i} \mathbf{x}_{i}, i=1, \ldots, r, r<n$. Let $C$ be an $r$-by-n matrix. Then $A+X C$ has spectrum

$$
\Gamma=\left\{\mu_{1}, \ldots, \mu_{r}, \lambda_{r+1}, \ldots, \lambda_{n}\right\},
$$

where $\mu_{1}, \ldots, \mu_{r}$ are eigenvalues of $\Omega+C X$, with $\Omega=\operatorname{diag}\left\{\lambda_{1}, \ldots, \lambda_{r}\right\}$.

The case $r=1$ in the Rado's result is the well known Brauer's perturbation result [3]. The following results will also be applied in this paper: Lemma 3.1 in [27], which shows how is the JCF of the Brauer perturbation, and Theorem 3.1 in [1], which gives a diagonalizable version of Rado's theorem.

Outline of the paper: the paper is organized as follows: In Section 2 and Section 3, with the diagonalizability that permit the Brauer Theorem and with the diagonalizability of circulant matrices, respectively, we characterize new lists of complex numbers in the left half-plane, which are UR. In Section 4, from the diagonalizable version of Rado's theorem, other left half-plane realizable lists are shown to be also UR. Finally, in Section 5 , we give new results to characterize more general lists of complex numbers being universally realizable. These results improve some previous universal realizability criteria. Examples are also given in each section.

\section{Spectra inhabiting the left half-plane that are universally realizable I}

It is clear that the diagonalizability of a realizing matrix is a necessary condition for the universal realizability. The diagonalizability condition is important because we know how to join blocks of a JCF to obtain blocks of bigger size. In general we cannot split up Jordan bloks. We may analyze the universal realizability from the point of view of diagonalizable realizability criteria, or from the point of view of diagonalizable realizing matrices. Criteria that were initially generated for a solution to RNIEP, later it was proved that they were also criteria for a solution to SNIEP, that is, for diagonalizable realizability. In [8], Ellard and Šmigoc proved that the following symmetric realizability criteria: $\mathcal{H}$-criterion by Ellard and Šmigoc [8], Soules criterion by Soules [32], $C$-realizability criterion by Borobia, Moro and Soto [2] and Family Soto- $p$ criteria by Soto [28], are all equivalent and contain all previously known symmetric criteria in the literature, except the criterion in [26]. From the point of view of realizing matrices we may consider normal, circulant, symmetric, bisymmetric and other diagonalizable matrices. In this section we shall use the diagonalizability that permit the Brauer Theorem.

Theorem 2.1. Let $\Lambda=\left\{\lambda_{1}, \lambda_{2}, \ldots, \lambda_{n}\right\}$ be a left half-plane list. Suppose that $\lambda_{2}, \ldots, \lambda_{r}$ are negative real numbers, $\lambda_{1}$ simple, and $\lambda_{r+1}, \ldots, \lambda_{n}$ are conjugate complex numbers with $\operatorname{Re} \lambda_{r+i}<0, \operatorname{Im} \lambda_{r+i}>0, i=1, \ldots n-$ r. If

$$
\lambda_{1} \geq-\sum_{k=2}^{r} \lambda_{k}+\sum_{j=1}^{\frac{n-r}{2}} S_{j}+T,
$$

where $S_{j}=-2 \operatorname{Re} \lambda_{r+2 j-1}$ if $-\operatorname{Re} \lambda_{r+2 j-1} \geq \operatorname{Im} \lambda_{r+2 j-1}$, with strict inequality in (1) if $-\operatorname{Re} \lambda_{r+2 j-1}=\operatorname{Im} \lambda_{r+2 j-1}$; or $S_{j}=-\operatorname{Re} \lambda_{r+2 j-1}+\operatorname{Im} \lambda_{r+2 j-1}$ if $-\operatorname{Re} \lambda_{r+2 j-1}<\operatorname{Im} \lambda_{r+2 j-1} ;$ and $T=\max _{1 \leq j \leq \frac{n-r}{2}} T_{j}$ for $T_{j}>0$ with $T_{j}=\operatorname{Re} \lambda_{r+2 j-1}+$ $\operatorname{Im} \lambda_{r+2 j-1}, j=1,2, \ldots, \frac{n-r}{2}$, then $\Lambda$ is universally realizable. 
Proof. Let

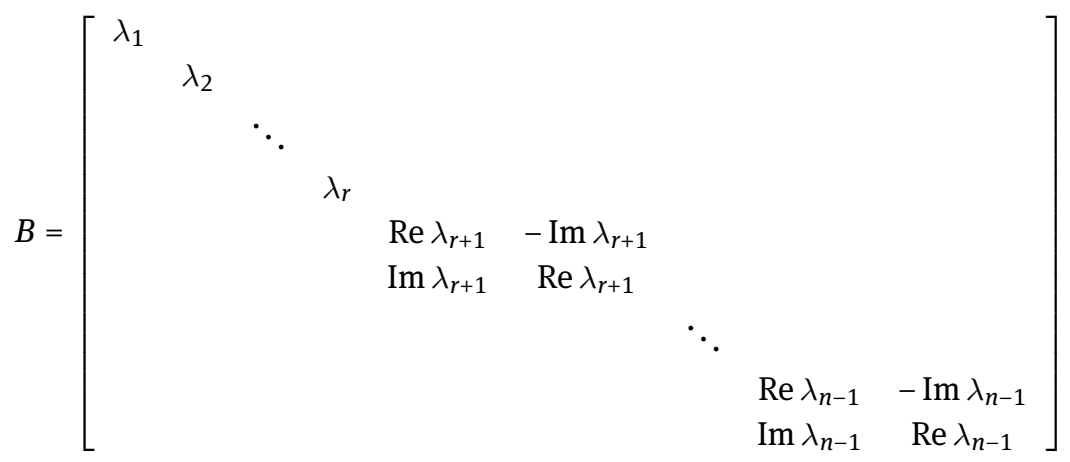

and $\lambda=-\sum_{k=2}^{r} \lambda_{k}-\sum_{j=1}^{\frac{n-r}{2}} S_{j}-T$ be with $S_{j}$ and $T$ as defined in (1). We shall apply Brauer's Theorem. Observe that $S_{j}$ is the amount we need to make nonnegative the 2-by-2 block

$$
\left[\begin{array}{cc}
\operatorname{Re} \lambda_{r+i} & -\operatorname{Im} \lambda_{r+i} \\
\operatorname{Im} \lambda_{r+i} & \operatorname{Re} \lambda_{r+i}
\end{array}\right], i=1,2, \ldots, n-r-1,
$$

$T=\max _{1 \leq j \leq \frac{n-r}{2}} T_{j}$ for $T_{j}>0$ is the amount we need to guarantee a nonnegative first column for the Brauer perturbation $A=B+\mathbf{e q}^{T}$, where $\mathbf{e}^{T}=[1,1, \ldots, 1]$. If $\max _{1 \leq j \leq \frac{n-r}{2}} T_{j} \leq 0$ we take $T=0$. Then

i) For

$$
\mathbf{q}^{T}=\left[\lambda,-\lambda_{2}, \ldots,-\lambda_{r},-\operatorname{Re} \lambda_{r+1}, h_{r+1}, \ldots,-\operatorname{Re} \lambda_{n-1}, h_{n-1}\right],
$$

where $h_{r+i}=-\operatorname{Re} \lambda_{r+i}$ if $-\operatorname{Re} \lambda_{r+i} \geq \operatorname{Im} \lambda_{r+i}$ or $h_{r+i}=\operatorname{Im} \lambda_{r+i}$ if $-\operatorname{Re} \lambda_{r+i}<\operatorname{Im} \lambda_{r+i}, A=B+\mathbf{e q}^{T}$ is diagonalizable nonnegative with spectrum $\Lambda$ and since $\operatorname{Re} \lambda_{r+i}<0, \operatorname{Im} \lambda_{r+i}>0, i=1, \ldots n-r, A$ has its first row being positive.

ii) If $-\operatorname{Re} \lambda_{r+i}=\operatorname{Im} \lambda_{r+i}$ with strict inequality in (1), then for the same $\mathbf{q}^{T}$ as in (2), $A=B+\mathbf{e q}^{T}$ is diagonalizable nonnegative with spectrum $\Lambda$ and with its first row being positive.

Thus, in both cases $A^{T}$ is cospectral to a diagonalizable nonnegative matrix with constant row sums and with its first column being positive. Then, from [5] it is similar to a positive matrix and therefore $\Lambda$ is universally realizable. Observe that if $-\operatorname{Re} \lambda_{r+i}>\operatorname{Im} \lambda_{r+i}$, for all $i \in[1, n-r-1], A$ is obtained diagonalizable ODP with spectrum $\Lambda$. Therefore, from [12], $\Lambda$ is also universally realizable.

Example 2.1. The list $\Lambda=\{10,-1,-2,-1+2 i,-1-2 i,-1+2 i,-1-2 i\}$ is diagonalizably realizable by the matrix

$$
\begin{aligned}
B= & {\left[\begin{array}{ccccccc}
10 & 0 & 0 & 0 & 0 & 0 & 0 \\
11 & -1 & 0 & 0 & 0 & 0 & 0 \\
12 & 0 & -2 & 0 & 0 & 0 & 0 \\
13 & 0 & 0 & -1 & -2 & 0 & 0 \\
9 & 0 & 0 & 2 & -1 & 0 & 0 \\
13 & 0 & 0 & 0 & 0 & -1 & -2 \\
9 & 0 & 0 & 0 & 0 & 2 & -1
\end{array}\right]+\left[\begin{array}{l}
1 \\
1 \\
1 \\
1 \\
1 \\
1 \\
1
\end{array}\right]\left[\begin{array}{ccccccc}
-9 & 1 & 2 & 1 & 2 & 1 & 2
\end{array}\right] } \\
& =\left[\begin{array}{ccccccc}
1 & 1 & 2 & 1 & 2 & 1 & 2 \\
2 & 0 & 2 & 1 & 2 & 1 & 2 \\
3 & 1 & 0 & 1 & 2 & 1 & 2 \\
4 & 1 & 2 & 0 & 0 & 1 & 2 \\
0 & 1 & 2 & 3 & 1 & 1 & 2 \\
4 & 1 & 2 & 1 & 2 & 0 & 0 \\
0 & 1 & 2 & 1 & 2 & 3 & 1
\end{array}\right] \in \mathcal{S}_{10} .
\end{aligned}
$$

Now, we take $B^{T}$, which has a positive Perron eigenvector

$$
\mathbf{x}^{T}=\left[\begin{array}{lllllll}
\frac{301}{264} & \frac{25}{44} & \frac{25}{44} & \frac{3}{4} & 1 & \frac{3}{4} & 1
\end{array}\right]
$$


and therefore $B^{T}$ is similar to the matrix

$$
A=D^{-1} B^{T} D=\left[\begin{array}{ccccccc}
1 & \frac{300}{301} & \frac{825}{301} & \frac{792}{301} & 0 & \frac{792}{301} & 0 \\
\frac{301}{150} & 0 & \frac{11}{6} & \frac{33}{25} & \frac{44}{25} & \frac{33}{25} & \frac{44}{25} \\
\frac{602}{275} & \frac{12}{11} & 0 & \frac{36}{25} & \frac{48}{25} & \frac{36}{25} & \frac{48}{25} \\
\frac{301}{198} & \frac{25}{33} & \frac{25}{18} & 0 & 4 & 1 & \frac{4}{3} \\
\frac{301}{132} & \frac{25}{22} & \frac{25}{12} & 0 & 1 & \frac{3}{2} & 2 \\
\frac{301}{198} & \frac{25}{33} & \frac{25}{18} & 1 & \frac{4}{3} & 0 & 4 \\
\frac{301}{132} & \frac{25}{22} & \frac{25}{12} & \frac{3}{2} & 2 & 0 & 1
\end{array}\right] \in \mathrm{CS}_{10}
$$

with its first column being positive, where $D=\operatorname{diag}\left\{x_{1}, \ldots, x_{n}\right\}$, with $x_{i} \in \mathbf{x}^{T}$. Then for

$$
\begin{gathered}
\mathbf{q}^{T}=\left[\begin{array}{ccccccc}
-\frac{6}{7} & \frac{1}{7} & \frac{1}{7} & \frac{1}{7} & \frac{1}{7} & \frac{1}{7} & \frac{1}{7}
\end{array}\right], \\
A+\mathbf{e q}^{T}=\left[\begin{array}{ccccccc}
\frac{1}{7} & \frac{49}{43} & \frac{124}{43} & \frac{835}{301} & \frac{1}{7} & \frac{835}{301} & \frac{1}{7} \\
\frac{1207}{1050} & \frac{1}{7} & \frac{83}{42} & \frac{256}{175} & \frac{333}{175} & \frac{256}{175} & \frac{333}{175} \\
\frac{2564}{1925} & \frac{95}{77} & \frac{1}{7} & \frac{277}{175} & \frac{361}{175} & \frac{277}{175} & \frac{361}{175} \\
\frac{919}{1386} & \frac{208}{231} & \frac{193}{126} & \frac{1}{7} & \frac{29}{7} & \frac{8}{7} & \frac{31}{21} \\
\frac{1315}{924} & \frac{197}{154} & \frac{187}{84} & \frac{1}{7} & \frac{8}{7} & \frac{23}{14} & \frac{15}{7} \\
\frac{919}{1386} & \frac{208}{231} & \frac{193}{126} & \frac{8}{7} & \frac{31}{21} & \frac{1}{7} & \frac{29}{7} \\
\frac{1315}{924} & \frac{197}{154} & \frac{187}{84} & \frac{23}{14} & \frac{15}{7} & \frac{1}{7} & \frac{8}{7}
\end{array}\right]
\end{gathered}
$$

is positive with spectrum $\Lambda$, and from [27, Lemma 3.1] it is also diagonalizable. Thus, $\Lambda$ is universally realizable.

Remark 2.1. Observe that from Theorem 2.1, to decide about the universal realizability of a list $\Lambda$ of complex numbers, we do not need to compute a nonnegative matrix with spectrum $\Lambda$ for each JCF allowed by $\Lambda$.

\section{Spectra inhabiting the left half-plane that are universally realizable II}

An ODP matrix is a nonnegative matrix with all its off-diagonal entries being positive (zero entries are permitted on the main diagonal), and a quasi-ODP matrix is an ODP matrix which admits one off-diagonal zero entry. Recall that in [12] the authors prove that if a list of complex numbers $\Lambda=\left\{\lambda_{1}, \lambda_{2}, \ldots, \lambda_{n}\right\}$ is diagonalizably ODP realizable, or it is diagonalizably quasi-ODP realizable, then $\Lambda$ is UR. Since the diagonalizability of a realizing matrix is necessary for universal realizability, here we shall consider as possible realizing matrices those that are circulant. Recall that an $n$-by- $n$ circulant matrix (see [25] and the classic book by Davis [6] ) is of the form

$$
C=\left[\begin{array}{cccccc}
c_{0} & c_{1} & c_{2} & \cdots & c_{n-2} & c_{n-1} \\
c_{n-1} & c_{0} & c_{1} & \ddots & \ddots & c_{n-2} \\
c_{n-2} & c_{n-1} & \ddots & \ddots & \ddots & \vdots \\
\vdots & \ddots & \ddots & \ddots & \ddots & c_{2} \\
c_{2} & \ddots & \ddots & \ddots & \ddots & c_{1} \\
c_{1} & c_{2} & \cdots & c_{n-2} & c_{n-1} & c_{0}
\end{array}\right],
$$

and it is uniquely determined by the entries of its first row. If $C$ is a real circulant matrix with $\operatorname{spectrum} \Lambda=$ $\left\{\lambda_{1}, \lambda_{2}, \ldots, \lambda_{n}\right\}$, then

$$
\lambda_{n-j+2}=\overline{\lambda_{j}}, j=2,3, \ldots, \frac{n+1}{2}
$$

and $\boldsymbol{\lambda}=\left[\lambda_{1}, \lambda_{2}, \ldots, \lambda_{n}\right]$ is said to be a conjugate-even vector. 
The explicit formulas for the $c_{k}$ are:

$$
c_{k}=\frac{1}{n}\left(\lambda_{1}+2 \sum_{j=2}^{m+1} \operatorname{Re} \lambda_{j} \cos \frac{2 k(j-1) \pi}{2 m+1}+2 \sum_{j=2}^{m+1} \operatorname{Im} \lambda_{j} \sin \frac{2 k(j-1) \pi}{2 m+1}\right),
$$

$k=0,1, \ldots, 2 m$ if $n=2 m+1$, and

$$
c_{k}=\frac{1}{n}\left(\lambda_{1}+2 \sum_{j=2}^{m+1} \operatorname{Re} \lambda_{j} \cos \frac{k(j-1) \pi}{m+1}+(-1)^{k} \lambda_{m+2}+2 \sum_{j=2}^{m+1} \operatorname{Im} \lambda_{j} \sin \frac{k(j-1) \pi}{m+1}\right),
$$

$k=0,1, \ldots, 2 m+1$ if $n=2 m+2$.

Theorem 3.1. Let $n=2 m+1$ and

$$
\Lambda=\left\{\lambda_{1}, \lambda_{2}, \ldots, \lambda_{m}, a+b i, a-b i, \lambda_{m}, \ldots, \lambda_{2}\right\}
$$

be a realizable zero trace list on the left half-plane, that is, $\lambda_{2}, \ldots, \lambda_{m}, a<0$ and

$$
\lambda_{1}=-2 a-2 \lambda_{2}-\cdots-2 \lambda_{m} .
$$

If

$$
-b \sin \frac{2 k m}{n} \pi<\sum_{j=2}^{m+1}\left(\cos \frac{2 k(j-1)}{n} \pi-1\right) \operatorname{Re} \lambda_{j}
$$

for $\theta=\frac{2 k m}{n} \pi$ in quadrant III or IV, then $\Lambda$ is the spectrum of an $n$-by-n circulant ODP matrix and therefore $\Lambda$ is universally realizable.

Proof. Since $\Lambda$ is a conjugate-even vector, we are going to show that $\Lambda$ is realizable by a circulant ODP matrix and therefore $\Lambda$ is UR. It is clear, from (5), that $c_{0}=0$. To show the positivity of $c_{k}, k=1, \ldots, n-1$, we observe that (3) can be written as

$$
c_{k}=\frac{1}{n}\left(2 \sum_{j=2}^{m+1}\left(\cos \frac{2 k(j-1) \pi}{n}-1\right) \operatorname{Re} \lambda_{j}+2 b \sin \frac{2 k m \pi}{n}\right) .
$$

Since $\cos \frac{2 k(j-1) \pi}{n}<1$ and $\operatorname{Re} \lambda_{j}<0,\left(\cos \frac{2 k(j-1) \pi}{n}-1\right) \operatorname{Re} \lambda_{j}>0$. Moreover if $\theta=\frac{2 k m \pi}{n}$ is located in quadrants I or II, then $\sin \theta \geq 0$ and the sum in (7) is also positive. Observe that $\operatorname{Im} \lambda_{j}=0$ for $j=2, \ldots, m$. If $\theta$ is in quadrants III or IV $(\sin \theta<0)$, then the condition (6) guarantees that the corresponding $c_{k}$ are also positive.

Theorem 3.2. Let $n=2 m+2$ and

$$
\Lambda=\left\{\lambda_{1}, \lambda_{2}, \ldots, \lambda_{m}, a+b i, \lambda_{m+2}, a-b i, \lambda_{m}, \ldots, \lambda_{2}\right\}
$$

be a realizable zero trace list on the left half-plane, that is, with

$$
\lambda_{2}, \ldots, \lambda_{m}, a, \lambda_{m+2}<0
$$

and

$$
\lambda_{1}=-2 a-2 \lambda_{2}-\cdots-2 \lambda_{m}-\lambda_{m+2} .
$$

If

$$
-b \sin \frac{k m \pi}{m+1}<\sum_{j=2}^{m+1}\left(\cos \frac{k(j-1) \pi}{m+1} \operatorname{Re} \lambda_{j}+\frac{1}{2}\left(-\lambda_{m+2}+(-1)^{k} \lambda_{m+2}\right)\right.
$$

for $\theta=\frac{k m}{m+1} \pi$ in quadrant III or IV, then $\Lambda$ is the spectrum of an $n$-by-n circulant ODP matrix and therefore $\Lambda$ is universally realizable.

Proof. It is clear, from (8), that $c_{0}=0$. To show the positivity of $c_{k}, k=1, \ldots, n-1$, we observe that (4) can be written as

$$
c_{k}=\frac{1}{n}\left(2 \sum_{j=2}^{m+1}\left(\cos \frac{k(j-1) \pi}{m+1}-1\right) \operatorname{Re} \lambda_{j}-\lambda_{m+2}+(-1)^{k} \lambda_{m+2}+2 b \sin \frac{k m \pi}{m+1}\right) .
$$


Since $\cos \frac{k(j-1) \pi}{m+1}<1$ and $\operatorname{Re} \lambda_{j}<0,\left(\cos \frac{k(j-1) \pi}{m+1}-1\right) \operatorname{Re} \lambda_{j}>0$. Obseve that $-\lambda_{m+2}+(-1)^{k} \lambda_{m+2} \geq 0$. Moreover if $\theta=\frac{k m \pi}{m+1}$ is located in quadrants I or II, then $\sin \theta \geq 0$ and the sum in (10) is also positive. Observe that $\operatorname{Im} \lambda_{j}=0$ for $j=2, \ldots, m$. If $\theta$ is in quadrant III or IV ( $\left.\sin \theta<0\right)$, then by the condition (9), the corresponding $c_{k}$ is also positive.

Example 3.1. Consider the list

$$
\Lambda=\{26,-4,-10,-10,-1+11 i,-1-11 i\}
$$

which can be ordered as the conjugate-even vector

$$
\lambda=[26,-10,-1+11 i,-4,-1-11 i,-10] .
$$

Then, by applying Theorem 3.2 we may compute the ODP circulant matrix

$$
\left[\begin{array}{cccccc}
0 & \frac{11}{6} \sqrt{3}+\frac{7}{2} & \frac{11}{2}-\frac{11}{6} \sqrt{3} & 8 & \frac{11}{6} \sqrt{3}+\frac{11}{2} & \frac{7}{2}-\frac{11}{6} \sqrt{3} \\
\frac{7}{2}-\frac{11}{6} \sqrt{3} & 0 & \frac{11}{6} \sqrt{3}+\frac{7}{2} & \frac{11}{2}-\frac{11}{6} \sqrt{3} & 8 & \frac{11}{6} \sqrt{3}+\frac{11}{2} \\
\frac{11}{6} \sqrt{3}+\frac{11}{2} & \frac{7}{2}-\frac{11}{6} \sqrt{3} & 0 & \frac{11}{6} \sqrt{3}+\frac{7}{2} & \frac{11}{2}-\frac{11}{6} \sqrt{3} & 8 \\
8 & \frac{11}{6} \sqrt{3}+\frac{11}{2} & \frac{7}{2}-\frac{11}{6} \sqrt{3} & 0 & \frac{11}{6} \sqrt{3}+\frac{7}{2} & \frac{11}{2}-\frac{11}{6} \sqrt{3} \\
\frac{11}{2}-\frac{11}{6} \sqrt{3} & 8 & \frac{11}{6} \sqrt{3}+\frac{11}{2} & \frac{7}{2}-\frac{11}{6} \sqrt{3} & 0 & \frac{11}{6} \sqrt{3}+\frac{7}{2} \\
\frac{11}{6} \sqrt{3}+\frac{7}{2} & \frac{11}{2}-\frac{11}{6} \sqrt{3} & 8 & \frac{11}{6} \sqrt{3}+\frac{11}{2} & \frac{7}{2}-\frac{11}{6} \sqrt{3} & 0
\end{array}\right]
$$

with spectrum $\Lambda$. Therefore $\Lambda$ is $U R$.

Example 3.2. Consider the list

$$
\Lambda=\{18,-3,-3,-3+9 i,-3-9 i,-3,-3\}
$$

Then, by applying Theorem 3.1 we may compute, to four decimal places, the ODP circulant matrix

$\left[\begin{array}{ccccccc}0 & 4.1157 & 0.98955 & 5.5069 & 0.49304 & 5.0104 & 1.8843 \\ 1.8843 & 0 & 4.1157 & 0.98955 & 5.5069 & 0.49304 & 5.0104 \\ 5.0104 & 1.8843 & 0 & 4.1157 & 0.98955 & 5.5069 & 0.49304 \\ 0.49304 & 5.0104 & 1.8843 & 0 & 4.1157 & 0.98955 & 5.5069 \\ 5.5069 & 0.49304 & 5.0104 & 1.8843 & 0 & 4.1157 & 0.98955 \\ 0.98955 & 5.5069 & 0.49304 & 5.0104 & 1.8843 & 0 & 4.1157 \\ 4.1157 & 0.98955 & 5.5069 & 0.49304 & 5.0104 & 1.8843 & 0\end{array}\right]$

with spectrum $\Lambda$. Therefore $\Lambda$ is $U R$.

Theorems 3.1 and 3.2 can be generalized to a finite number of pairs of conjugate complex numbers as follows:

Theorem 3.3. Let $n=2 m+1$ and

$$
\Lambda=\left\{\lambda_{1}, \lambda_{2}, \ldots, \lambda_{s}, a+b i, \ldots, a+b i, a-b i, \ldots, a-b i, \lambda_{s}, \ldots, \lambda_{2}\right\}
$$

be a realizable zero trace list on the left half-plane, that is, $\lambda_{2}, \ldots, \lambda_{s}, a<0$ and

$$
\lambda_{1}=-2(m+1-s) a-2 \lambda_{2}-\cdots-2 \lambda_{s}
$$

If

$$
-b\left(\sum_{j=S+1}^{m+1} \sin \frac{2 k(j-1) \pi}{n}\right)<\sum_{j=2}^{m+1}\left(\cos \frac{2 k(j-1)}{n} \pi-1\right) \operatorname{Re} \lambda_{j}
$$

for $\theta=\frac{2 k(j-1)}{n} \pi$ in quadrant III or IV, then $\Lambda$ is the spectrum of an $n$-by-n circulant ODP matrix and therefore $\Lambda$ is universally realizable. 
Proof. The proof follows as the proof of Theorem 3.1.

Theorem 3.4. Let $n=2 m+2$ and

$$
\Lambda=\left\{\lambda_{1}, \lambda_{2}, \ldots, \lambda_{s}, a+b i, \ldots, a+b i, \lambda_{m+2}, a-b i, \ldots, a-b i, \lambda_{s}, \ldots, \lambda_{2}\right\}
$$

be a realizable zero trace list on the left half-plane, that is, with

$$
\lambda_{2}, \ldots, \lambda_{s}, a, \lambda_{m+2}<0
$$

and

$$
\lambda_{1}=-2(m+1-s) a-2 \lambda_{2}-\cdots-2 \lambda_{s}-\lambda_{m+2}
$$

If

$$
-b\left(\sum_{j=s+1}^{m+1} \sin \frac{k(j-1) \pi}{m+1}\right)<\sum_{j=2}^{m+1}\left(\cos \frac{k(j-1) \pi}{m+1} \operatorname{Re} \lambda_{j}+\frac{1}{2}\left(-\lambda_{m+2}+(-1)^{k} \lambda_{m+2}\right)\right.
$$

for $\theta=\frac{k(j-1)}{m+1} \pi$ in quadrant III or IV, the corresponding $c_{k}$ is positive. Then $\Lambda$ is the spectrum of an $n$-by- $n$ circulant ODP matrix and therefore $\Lambda$ is universally realizable.

Proof. The proof follows as the proof of Theorem 3.2.

Observe that from theorems in this section, to decide on the universal realizability of a conjugate-even list $\Lambda$ of complex numbers, it is enough to show that $\Lambda$ is realizable by a ODP circulant matrix.

\section{Spectra inhabiting the left half plane that are universally realizable III}

Rados's result (here Theorem 1.1) has been important to generate many of the known sufficient conditions in the literature for both problems, the NIEP and the URP, to have a solution. A number of distinct versions of Rado's result, to be applied to different problems, have also been obtained. In [1] the authors prove a diagonalizable version of the Rado's Theorem, which allows us in this section, to generate more general left half-plane lists that are UR.

Theorem 4.1. Let $\Lambda=\left\{\lambda_{1}, \lambda_{2}, \ldots, \lambda_{n}\right\}$ be a list of complex numbers with

$$
\Lambda=\bar{\Lambda}, \lambda_{1} \geq \max _{i}\left|\lambda_{i}\right|, \sum_{i=1}^{n} \lambda_{i} \geq 0,
$$

Suppose that:

i) $\Lambda=\Lambda_{0} \cup \Lambda_{1} \cup \cdots \cup \Lambda_{p_{0}}$, with

$$
\begin{aligned}
& \Lambda_{0}=\left\{\lambda_{01}, \lambda_{02}, \ldots, \lambda_{0 p_{0}}\right\}, \quad \lambda_{1}=\lambda_{01}, \\
& \Lambda_{k}=\left\{\lambda_{k 1}, \lambda_{k 2}, \ldots, \lambda_{k p_{k}}\right\}, \quad k=1, \ldots, p_{0},
\end{aligned}
$$

where some lists $\Lambda_{k}$ can be empty, and for each $k=1, \ldots p_{0}, \Gamma_{k}=\left\{\omega_{k}\right\} \cup \Lambda_{k}, 0 \leq \omega_{k} \leq \lambda_{1}$, is diagonalizably realizable by a matrix $A_{k} \in \mathcal{C} \mathcal{S}_{\omega_{k}}$. Moreover, $A=\operatorname{diag}\left\{A_{1}, A_{2}, \ldots, A_{p_{0}}\right\}$ has a diagonal JCF $J(A)=S^{-1} A S$, where $S=[X \mid Y], S^{-1}=\left[\begin{array}{c}\widetilde{X} \\ \widetilde{Y}\end{array}\right]$.

ii) There is a $p_{0}$-by- $p_{0}$ diagonalizable nonnegative matrix $B$ with spectrum $\Lambda_{0}$, diagonal entries $\omega_{1}, \omega_{2}, \ldots, \omega_{p_{0}}$ and a positive column. Let $C=B-\Omega$ with $\Omega=\operatorname{diag}\left\{\omega_{1}, \omega_{2}, \ldots, \omega_{p_{0}}\right\}$.

Then, $A+X C \widetilde{X}$ is diagonalizable nonnegative with a positive column or it is a diagonalizable ODP matrix. Hence, $\Lambda$ is universally realizable. 
Proof. From i) $A=\operatorname{diag}\left\{A_{1}, A_{2}, \ldots, A_{p_{0}}\right\}$ is an $n$-by- $n$ diagonalizable nonnegative matrix realizing $\Gamma=\Gamma_{1} \cup$ $\cdots \cup \Gamma_{p_{0}}$. where $\Gamma_{i}$ is the spectrum of $A_{i}$.

From ii) since $S^{-1} S=I_{n}$, then $\widetilde{X} X=I_{r}, \widetilde{X} Y=0, \widetilde{Y} X=0, \widetilde{Y} Y=I_{n-r}$. As $A_{k} \in \mathcal{C} \mathcal{S}_{\omega_{k}}, X$ will be nonnegative and since $B$ has a positive column, $A+X C \widetilde{X}$ will also have a positive column. Then, from Rado's diagonalizable version [1, Theorem 3.1], $A+X C \widetilde{X}$ is diagonalizable nonnegative with spectrum $\Lambda$ and a positive column or it is a diagonalizable ODP matrix.

Hence from [5] or from [12], $\Lambda$ is also UR.

Example 4.1. Consider the spectrum

$$
\begin{aligned}
\Lambda & =\{13,-1,-2+5 i,-2-5 i,-2+5 i,-2-5 i\}, \text { with } \\
\Lambda & =\Lambda_{0} \cup \Lambda_{1} \cup \Lambda_{2} \cup \Lambda_{3}, \text { where } \\
\Lambda_{0} & =\{13,-2+5 i,-2-5 i\}, \Lambda_{1}=\{-2+5 i,-2-5 i\}, \\
\Lambda_{2} & =\{-1\}, \Lambda_{3}=\emptyset \\
\Gamma_{1} & =\{7,-2+5 i,-2-5 i\}, \quad \Gamma_{2}=\{1,-1\}, \Gamma_{3}=\{1\} .
\end{aligned}
$$

Then the matrices

$$
A_{1}=\left[\begin{array}{ccc}
1 & 6 & 0 \\
0 & 1 & 6 \\
\frac{17}{3} & \frac{1}{3} & 1
\end{array}\right], A_{2}=\left[\begin{array}{ll}
0 & 1 \\
1 & 0
\end{array}\right], A_{3}=[1]
$$

are diagonalizable realizing $\Gamma_{1}, \Gamma_{2}$ and $\Gamma_{3}$, respectively. Moreover

$$
B=\left[\begin{array}{ccc}
7 & 0 & 6 \\
\frac{53}{6} & 1 & \frac{19}{6} \\
0 & 12 & 1
\end{array}\right] \text {, with } C=\left[\begin{array}{ccc}
0 & 0 & 6 \\
\frac{53}{6} & 0 & \frac{19}{6} \\
0 & 12 & 0
\end{array}\right] \text {, }
$$

is diagonalizable nonnegative with spectrum $\Lambda_{0}$ and with the required diagonal entries. Then,

$$
\begin{aligned}
& A+X C \widetilde{X}= \\
& {\left[\begin{array}{cccccc}
1 & 6 & 0 & 0 & 0 & 0 \\
0 & 1 & 6 & 0 & 0 & 0 \\
\frac{17}{3} & \frac{1}{3} & 1 & 0 & 0 & 0 \\
0 & 0 & 0 & 0 & 1 & 0 \\
0 & 0 & 0 & 1 & 0 & 0 \\
0 & 0 & 0 & 0 & 0 & 1
\end{array}\right]+\left[\begin{array}{ccc}
1 & 0 & 0 \\
1 & 0 & 0 \\
1 & 0 & 0 \\
0 & 1 & 0 \\
0 & 1 & 0 \\
0 & 0 & 1
\end{array}\right]\left[\begin{array}{ccc}
0 & 0 & 6 \\
\frac{53}{6} & 0 & \frac{19}{6} \\
0 & 12 & 0
\end{array}\right]\left[\begin{array}{cccccc}
\frac{17}{53} & \frac{18}{53} & \frac{18}{53} & 0 & 0 & 0 \\
0 & 0 & 0 & \frac{1}{2} & \frac{1}{2} & 0 \\
0 & 0 & 0 & 0 & 0 & 1
\end{array}\right]} \\
& =\left[\begin{array}{cccccc}
1 & 6 & 0 & 0 & 0 & 6 \\
0 & 1 & 6 & 0 & 0 & 6 \\
\frac{17}{3} & \frac{1}{3} & 1 & 0 & 0 & 6 \\
\frac{17}{6} & 3 & 3 & 0 & 1 & \frac{19}{6} \\
\frac{17}{6} & 3 & 3 & 1 & 0 & \frac{19}{6} \\
0 & 0 & 0 & 6 & 6 & 1
\end{array}\right] \in S_{13}
\end{aligned}
$$

is diagonalizable nonnegative with spectrum $\Lambda$ and with a positive column. Thus, for $\mathbf{q}^{T}=$ $\left[\begin{array}{llllll}\frac{1}{5} & \frac{1}{5} & \frac{1}{5} & \frac{1}{5} & \frac{1}{5} & -1\end{array}\right], M=A+X C \widetilde{X}+\mathbf{e q}^{T}$ is a diagonalizable ODP matrix with spectrum $\Lambda$. Therefore $\Lambda$ is $U R$.

Remark 4.1. The list from example 2.1 satisfies conditions of Theorem 2.1, but it cannot be the spectrum of a circulant matrix. The spectra of the circulant matrices of examples 3.1 and 3.2 do not satisfy conditions of Theorem 2.1. Then criterion from Theorem 2.1 and criteria from Theorem 3.1 and Theorem 3.2 are independent. It is clear that criteria from Theorem 3.1 and Theorem 3.2 and criterion from Theorem 4.1 are also independent. However, by its own nature, criterion from Theorem 4.1 trivially contains criterion from Theorem 2.1. 


\section{More general spectra that are universally realizable}

In this section we apply the procedure in Section 4 to more general lists of complex numbers (not in the left half-plane). For instance, applying Rado's Theorem to the following 4 lists we have:

i) $\Lambda=\{8,6,3,3,-5,-5,-5,-5\}$, with $\Lambda_{0}=\{8,6\}, \Lambda_{1}=\Lambda_{2}=\{3,-5,-5\}$ and $\Gamma_{1}=\Gamma_{2}=\{7,3,-5,-5\}$, is diagonalizably ODP realizable and therefore it is UR.

ii) $\Lambda=\{7,5,1,1,-4,-4,-6\}$ with $\Lambda_{0}=\{7,5\}, \Gamma_{1}=\{6,1,1,-4,-4\}, \Gamma_{2}=\{6,-6\}$ is also diagonalizably ODP realizable and therefore it is UR.

iii) $\Lambda=\{10,3,2,-1,-1,3 i,-3 i,-1 \pm 2 i,-1 \pm 2 i\}$ with $\Lambda_{0}=\{10,3,2\}, \Gamma_{1}=\{6,3 i,-3 i\}, \Gamma_{2}=\Gamma_{3}=$ $\left\{\frac{9}{2},-1,-1 \pm 2 i\right\}$ is the spectrum of a diagonalizable nonnegative matrix $A \in \mathcal{C} S_{10}$ with a positive column. Hence it is also UR.

iv) $\Lambda=\{13,3,1+4 i, 1-4 i, 1+4 i, 1-4 i\}$ with $\Lambda_{0}=\{13,3\}, \Gamma_{1}=\Gamma_{2}=\{8,1+4 i, 1-4 i\}$ is the spectrum of a diagonalizable positive matrix. Hence it is also UR, etc.

One of the advantage of applying procedure in Section 4 is that to decide whether $\Lambda=\left\{\lambda_{1}, \lambda_{2}, \ldots, \lambda_{n}\right\}$ is UR we do not need to compute a nonnegative matrix for each JCF allowed by $\Lambda$. It is enough to show that $\Lambda$ is diagonalizably ODP realizable or $\Lambda$ is the spectrum of a diagonalizable nonnegative matrix with constant row sums and a positive row or column.

In [22], Minc shows that even if $\Lambda=\left\{1,-\frac{1}{2},-\frac{1}{2}\right\}$ is $\mathrm{UR}, \Lambda$ is not doubly stochastic universally realizable. That is, no $3 \times 3$ doubly stochastic matrix has a JCF with a Jordan block $J_{2}\left(-\frac{1}{2}\right)$. This shows that the answer to the corresponding question in the introduction is in the negative. However, in [31] the authors give sufficient conditions for the real permutative universal realizability problem has a solution in certain cases, that is, the problem of finding a permutative nonnegative matrix with real spectrum $\Lambda=\left\{\lambda_{1}, \lambda_{2}, \ldots, \lambda_{n}\right\}$ for each JCF allowed by $\Lambda$.

Given a list of complex numbers $\Lambda=\left\{\lambda_{1}, \lambda_{2}, \ldots, \lambda_{n}\right\}$, the procedure in Section 4 (Rado diagonalizable again) allows to construct a family of universally realizable spectra with a fixed sublist $\Lambda^{\prime} \subset \Lambda$ : Consider the lists

$$
\begin{aligned}
\Lambda=\{7,-3,-3,1+i, 1-i, 1+3 i, 1-3 i\} \\
\Lambda^{\prime}=\{-3,-3,1+i,-1-i\} \text { with } \\
\Lambda=\Lambda_{0} \cup \Lambda_{1} \cup \Lambda_{2} \cup \Lambda_{3} \\
\Lambda_{0}=\{7,1+3 i, 1-3 i\}, \Lambda_{1}=\Lambda_{2}=\{-3\}, \Lambda_{3}=\{1+i, 1-i\} \\
\Gamma_{1}=\Gamma_{2}=\{3,-3\}, \Gamma_{3}=\{3,1+i, 1-i\} .
\end{aligned}
$$

The diagonalizable matrices

$$
A_{1}=A_{2}=\left[\begin{array}{ll}
0 & 3 \\
3 & 0
\end{array}\right] \text { and } A_{3}=\left[\begin{array}{ccc}
\frac{5}{3} & \frac{2}{3}-\frac{1}{3} \sqrt{3} & \frac{1}{3} \sqrt{3}+\frac{2}{3} \\
\frac{1}{3} \sqrt{3}+\frac{2}{3} & \frac{5}{3} & \frac{2}{3}-\frac{1}{3} \sqrt{3} \\
\frac{2}{3}-\frac{1}{3} \sqrt{3} & \frac{1}{3} \sqrt{3}+\frac{2}{3} & \frac{5}{3}
\end{array}\right]
$$


realize $\Gamma_{1}, \Gamma_{2}$ and $\Gamma_{3}$, respectively. Moreover,

$$
B=\left[\begin{array}{ccc}
3 & 0 & 4 \\
\frac{13}{4} & 3 & \frac{3}{4} \\
0 & 4 & 3
\end{array}\right]
$$

has spectrum $\Lambda_{0}$ with diagonal entries 3, 3, 3 (the Perron eigenvalues of $A_{1}, A_{2}, A_{3}$ ). Then

$$
\left[\begin{array}{ccc}
A_{1} & & \\
& A_{2} & \\
& & A_{3}
\end{array}\right]+X C \widetilde{X}
$$

with

$$
C=B-\operatorname{diag}\{3,3,3\}=\left[\begin{array}{ccc}
0 & 0 & 4 \\
\frac{13}{4} & 0 & \frac{3}{4} \\
0 & 4 & 0
\end{array}\right]
$$

is a diagonalizable nonnegative matrix with spectrum $\Lambda=\Lambda_{0} \cup \Lambda^{\prime}$ and with a positive column. Therefore $\Lambda$ is UR. Now, if we change $\Lambda_{0}$ to $\Lambda_{0, a}=\{11,-1+3 i,-1-3 i\}$, with realizing matrix

$$
B=\left[\begin{array}{ccc}
3 & 0 & 8 \\
\frac{25}{8} & 3 & \frac{39}{8} \\
0 & 8 & 3
\end{array}\right]
$$

we obtain a diagonalizable nonnegative matrix with spectrum $\Lambda_{0, a} \cup \Lambda^{\prime}=\{11,-3,-3,1+i, 1-i,-1+3 i,-1-$ $3 i\}$ and with a positive column. Thus $\Lambda_{0, a} \cup \Lambda^{\prime}$ is also UR. Taking $\Lambda_{0, b}=\{13,-2+3 i,-2-3 i\}$ with realizing matrix

$$
B=\left[\begin{array}{ccc}
3 & 0 & 10 \\
\frac{17}{5} & 3 & \frac{33}{5} \\
0 & 10 & 3
\end{array}\right]
$$

we obtain that $\Lambda_{0, b} \cup \Lambda^{\prime}=\{13,-3,-3,1+i, 1-i,-2+3 i,-2-3 i\}$ is also UR, etc.

Next we condider spectra in the right half-plane, that is, complex spectra satisfying $\operatorname{Re} \lambda_{i} \geq 0, i=1,2, \ldots, n$.

Corollary 5.1. Let $\Lambda=\left\{\lambda_{1}, \lambda_{2}, \ldots, \lambda_{n}\right\}$ be a list of complex numbers in the right half-plane, where $\lambda_{1}, \lambda_{2}, \ldots, \lambda_{r}$ are positive real numbers, $\lambda_{1}$ simple, and $\lambda_{r+1}, \ldots, \lambda_{n}$ are conjugate complex numbers with $\operatorname{Re} \lambda_{r+j} \geq 0, \operatorname{Im} \lambda_{r+j}>0, j=1, \ldots n-r$. If

$$
\lambda_{1}>-\sum_{k=r+1, \operatorname{Im} \lambda_{k}<0}^{n} \operatorname{Im} \lambda_{k}+\max _{2 \leq j \leq n}\left\{\operatorname{Re} \lambda_{j}+\operatorname{Im} \lambda_{j}\right\},
$$

then $\Lambda$ is universally realizable.

Proof. It is enough to apply the Brauer's result to obtain a diagonalizable nonnegative matrix with its first column being positive. Observe that in many examples we may reach the universal realizability with $\lambda_{1}$ being smaller than the value in (15).

Example 5.1. Consider

$$
\begin{aligned}
\Lambda & =\{22,3,3,3,1 \pm 3 i, 1 \pm 3 i, 2 \pm 5 i, 2 \pm 5 i\} \text { with } \\
\Lambda_{0} & =\{22,2 \pm 5 i\}, \Gamma_{1}=\{12,2 \pm 5 i\}, \\
\Gamma_{2} & =\{10,1 \pm 3 i, 1 \pm 3 i\}\}, \Gamma_{3}=\{4,3,3,3\} .
\end{aligned}
$$

The diagonalizable matrices

$$
B=\left[\begin{array}{ccc}
12 & 0 & 10 \\
\frac{125}{18} & 10 & \frac{91}{18} \\
0 & 18 & 4
\end{array}\right], A_{1}=\left[\begin{array}{ccc}
7 & 0 & 5 \\
10 & 2 & 0 \\
0 & 5 & 7
\end{array}\right]
$$




$$
A_{2}=\left[\begin{array}{lllll}
4 & 0 & 3 & 0 & 3 \\
6 & 1 & 0 & 0 & 3 \\
0 & 3 & 4 & 0 & 3 \\
6 & 0 & 3 & 1 & 0 \\
0 & 0 & 3 & 3 & 4
\end{array}\right], A_{3}=\left[\begin{array}{llll}
3 & 0 & 0 & 1 \\
0 & 3 & 0 & 1 \\
0 & 0 & 3 & 1 \\
0 & 0 & 0 & 4
\end{array}\right]
$$

realize the spectrum $\Lambda_{0}, \Gamma_{1}, \Gamma_{2}, \Gamma_{3}$, respectively. Then, for

$$
C=\left[\begin{array}{ccc}
0 & 0 & 10 \\
\frac{125}{18} & 0 & \frac{91}{18} \\
0 & 18 & 0
\end{array}\right] \text {, the matrix }\left[\begin{array}{ccc}
A_{1} & & \\
& A_{2} & \\
& & A_{3}
\end{array}\right]+X C \tilde{X}
$$

becomes a diagonalizable nonnegative matrix with its last column being positive. Hence, $\Lambda$ is UR.

Acknowledgement: Supported by Universidad Católica del Norte-VRIDT 036-2020, Núcleo 6 UCN VRIDT083-2020, Chile.

Data Availability Statement: All data generated or analyzed during this study are included in this published article [and its supplementary information files].

\section{References}

[1] L. E. Arrieta, A. D. Millano, R. L. Soto, On spectra realizable and diagonalizably realizable, Linear Algebra Appl. 612 (2021) 273-288.

[2] A. Borobia, J. Moro, R. L. Soto, A unified view on compensation criteria in the real nonnegative inverse eigenvalue problem, Linear Algebra Appl. 428 (2008) 2574-2584.

[3] A. Brauer, Limits for the characteristic roots of a matrix IV. Applications to stochastic matrices, Duke Math. J. 19 (1952) 75-91.

[4] M. Collao, C. R. Johnson; R. L. Soto, Universal realizability of spectra with two positive eigenvalues, Linear Algebra Appl. 545 (2018) 226-239.

[5] M. Collao, M. Salas, R. L. Soto, Spectra universally realizable by doubly stochastic matrices, Special Matrices 6 (2018) 301309.

[6] P. J. Davis, Circulant Matrices, John Wiley \& sons, New York, 1979.

[7] R. C. Díaz, R. L. Soto, Nonnegative inverse elementary divisors problem in the left half-plane, Linear and Multilinear Algebra 64 (2016) 258-268.

[8] R. Ellard and H. Šmigoc, Connecting sufficient conditions for the symmetric nonnegative inverse eigenvalue problem, Linear Algebra Appl. 498 (2016) 521-552.

[9] C. R. Johnson, Row stochastic matrices similar to doubly stochastic matrices, Linear and Multilinear Algebra 10 (1981) 113130.

[10] C. R. Johnson, T. J. Laffey, R. Loewy, The real and symmetric nonnegative inverse eigenvalue problems are different, Proc. AMS 124 (1996) 3647-3651.

[11] C. R. Johnson, C. Marijuán, P. Paparella, M. Pisonero, The NIEP, Operator Theory: Advances and Applications 267 (2018) 199-220.

[12] C. R. Johnson, A. I. Julio, R. L. Soto, Nonnegative realizability with Jordan structure, Linear Algebra Appl. 587 (2020) 302-313.

[13] A. I. Julio, C. Marijuán, M. Pisonero, R. L. Soto, On universal realizability of spectra, Linear Algebra Appl. 563 (2019) $353-372$.

[14] A. I. Julio, R. L. Soto, On the universal realizability problem, Linear Algebra Appl. 597 (2020) 170-186.

[15] A. I. Julio, C. Marijuán, M. Pisonero, R. L. Soto, Universal realizability in low dimension, Linear Algebra Appl. 619 (2021) 107-136.

[16] F. Karpelevic, On the eigenvalues of a matrix with nonnegative elements, Izv. Akad. Nauk SSSR Ser. Mat. 15 (1951) 361 -383.

[17] A. N. Kolmogorov, Markov chains with a countable number of possible states, Bull Moskov Gosu-darstvennogo Univ. Mat. Meh. 1(3) (1937) 1-16.

[18] T. J. Laffey, M. E. Meehan, A characterization of trace zero nonnegtaive $5 \times 5$ matrices, Linear Algebra Appl. $302 / 303$ (1999) 295-302.

[19] R. Loewy, D. London, A note on an inverse problem for nonnegative matrices, Linear and Multilinear Algebra 6 (1978) 83-90.

[20] M. E. Meehan, Some results on matrix spectra, Ph. D. Thesis, National University of Ireland, dublin, 1998.

[21] H. Minc, Inverse elementary divisor problem for nonnegative matrices, Proc. of the Amer. Math. Society 83 (4) (1981) 665669. 
[22] H. Minc, Inverse elementary divisor problem for doubly stochastic matrices, Linear and Multilinear Algebra 11 (1982) $121-131$.

[23] H. Perfect, Methods of constructing certain stochastic matrices, Duke Math. J. 20 (1953) 395-404.

[24] H. Perfect, Methods of constructing certain stochastic matrices II, Duke Math. J. 22 (1955) 305-311.

[25] O. Rojo, R. L. Soto, Guo perturbation for symmetric nonnegative circulant matrices, Linear Algebra Appl. 431 (2009) 594-607.

[26] R. L. Soto, O. Rojo, J. Moro, A. Borobia, Symmetric nonnegative realization of spectra, Electronic J. Linear Algebra 16 (2007) 1-18

[27] R. L. Soto, J. Ccapa, Nonnegative matrices with prescribed elementary divisors, Electronic J. Linear Algebra 17 (2008) 287 303..

[28] R. L. Soto, A family of realizability criteria for the real and symmetric nonnegative inverse eigenvalue problem, Numer. Linear Algebra Appl. 20 (2013) 336-348.

[29] R. L. Soto, R. C. Díaz, H. Nina, M. Salas, Nonnegative matrices with prescribed spectrum and elementary divisors, Linear Algebra Appl. 439 (2013) 3591-3604.

[30] R. L. Soto, R. C. Díaz, M. Salas, O. Rojo, M-matrices with prescribed elementary divisors, Inverse Problems 33 (2017) 095009, https:/doi.org/10. 1088/1361-6420/aa7b91.

[31] R. L. Soto, A. I. Julio, J. H. Alfaro, Permutative universal realizability, Special Matrices 9 (2021) 66-77.

[32] G. Soules, Constructing symmetric nonnegative matrices, Linear and Multilinear Algebra 13 (1983) 241-251.

[33] 0. Spector, A characterization of trace zero symmetric $5 \times 5$ matrices, Linear Algebra Appl. 434 (2011) 1000-1017.

[34] H. R. Suleimanova, Stochastic matrices with real characteristic values, Dokl. Akad. Nauk SSSR 66 (1949) 343-345.

[35] J. Torre-Mayo, M. R. Abril-Raymundo, E. Alarcia-Estévez, C. Marijuán, M. Pisonero, The nonnegative inverse eigenvalue problem from the coefficients of the characteristic polynomial. EBL digraphs, Linear Algebra Appl. 426 (2007) 729-773. 